\title{
Calculation of constructive elements of mobile overpass
}

\author{
Adil Kadyrov, Aleksandr Ganyukov, Marat Imanov and Kyrmyzy Balabekova* \\ Karaganda State Technical University, Mira Boulevard, 56, Karaganda, 100000, Kazakhstan
}

\begin{abstract}
In this article we discuss the construction of modular mobile overcrossing. Calculation of its constructive elements is performed and the optimum length of one module is determined. The purpose of the study is to develop a technique and calculate constructive elements of mobile bridge overcrossing in order to reduce traffic jams. Mathematical analysis, finite elements, finite differences and analytical method of displacements have been used in this study. The optimum length of each module of bridge overcrossing is received. The solution matrix of the method of finite differences allows us to calculate carriageway plate of the overcrossing with a wide variety of geometrical sizes, and also in case of different flexural stiffness properties of the plate and reinforcing elements. Calculation of the spatial frame of bridge overcrossing is performed by precise analytical method of relocation taking into account the bend and torsion of its elements.
\end{abstract}

Keywords: Constructive elements, mathematical analysis, mobile overcrossing, traffic jam.

ALL major cities in the world are affected by traffic jams on roads, including twofold and threefold crossroads. This can be eliminated using various methods of traffic regulation, as well as construction of basic overcrossings of various heights and configurations.

Today in the largest cities of the world, there are several ways of preventing traffic jams, like building huge platforms, bridges and overpasses. Construction of overpasses demands free territory, which is not easily available at present. Thus, various folded constructions are done, e.g. the Jacques Shaban Delm Bridge in France, the Rog Bridge in Germany and folded bridges in Russia.

The analog of such constructions is the tank bridge layer which is, in fact, a folded mobile bridge. Here, we discuss the construction of a mobile overcrossing which can be quickly assembled on a crossroad during traffic blocks. The mobile overcrossing consists of horizontal modules equipped with a wheel mechanism and bracing jacks ${ }^{1}$.

In necessary cases modules are transported to the crossroads and connected by special fixing elements forming one construction. At the same time bracing jacks lean on

\footnotetext{
*For correspondence. (e-mail: 0603 92@mail.ru)
}

the basis. The mobile overcrossing is different from military bridge layers as it has to satisfy traffic rules: passing height under it is more than $4.5 \mathrm{~m}$ and transport strip width in one direction not less than $3.5 \mathrm{~m}$.

The mobile overcrossing has two main modules: sloping (Figure $1 a$ ) and horizontal (Figure $1 b$ ). As shown in Figure 1, the basic constructive elements of the overcrossing are: barrier (1); plate (2); support (3); wheel engine (4), and support and the mechanism of lifting it up/down (5). A patent has been received for the construction of the mobile overcrossing.

\section{Methods}

The assembled construction allows vehicles to cross the perpendicular road, where there is traffic block at the crossroads (Figure 2).

In the first stage there are two tasks: (i) Determination of optimum length of modules of the overcrossing taking into consideration the geometrical size of the carriageway; (ii) calculation and analysis of all elements of the construction overcrossing for durability, rigidity and stability.
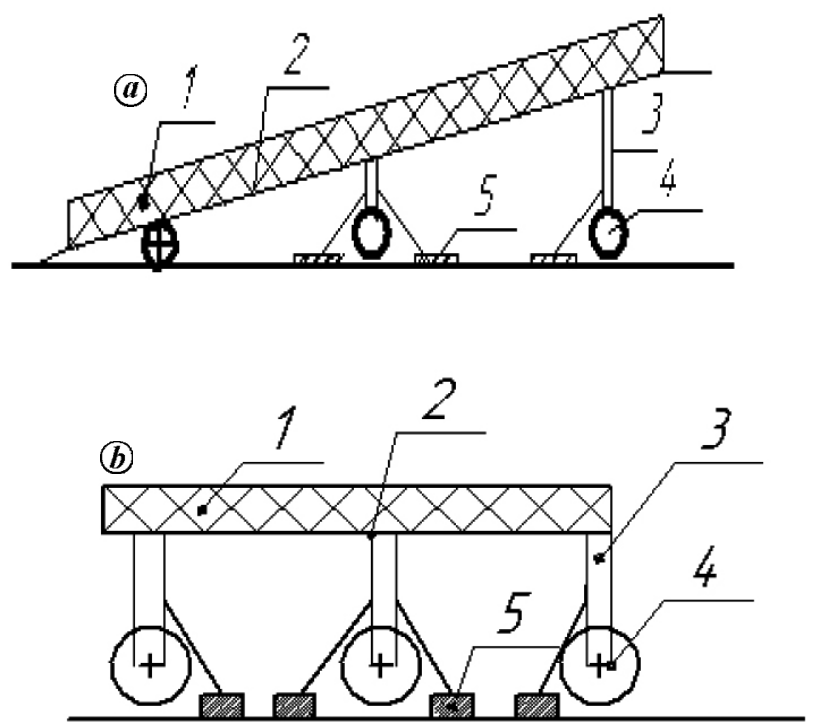

Figure 1. Modules of the overcrossing: $\boldsymbol{a}$, sloping module; $\boldsymbol{b}$, horizontal module. 


\section{Results}

In case of the first task, it is assumed that weight of the module is proportional to its length $x$, to the quantity of supports $n$, equipped with wheels for driving of the overcrossing and the weight of one support $q$. Then the total weight $G$ is determined as

$$
G=k x+\frac{L \times n}{x} \times q,
$$

where $k$ is the weight of the overcrossing per metre of length $(\mathrm{N} / \mathrm{m}), L$ the length of the overcrossing $(\mathrm{m}), n$ the number of supports, $q$ the weight of one support $(\mathrm{N})$ and $L / x$ is the number of modules.

The weight derivatives lengthwise of the overcrossing are presented as follows

$$
\begin{aligned}
& G^{\prime}=k x-\frac{L n q}{x^{2}}, \\
& G^{\prime \prime}=\frac{2 L n q}{x^{3}} .
\end{aligned}
$$

For the critical point (minimax point) at $G^{\prime}=0$,

$$
\begin{aligned}
& k=\frac{\operatorname{Lnq}}{x}, \\
& x_{\mathrm{cr}}=\sqrt{\frac{L n q}{k}} .
\end{aligned}
$$

As $G^{\prime \prime}$ is greater than zero, the point $x_{\text {cr }}$ determines the optimum length of one module from the condition of minimization of total load of the overcrossing.

For example, for an overcrossing $30 \mathrm{~m}$ long, with four supports on each module, and at each support weight $1.5 \times 10^{4} \mathrm{~N}$ and $3 \times 10^{4} \mathrm{~N}$ per metre length of the construction, the optimum length $(\mathrm{m})$ will be

$$
x_{\kappa p}=\sqrt{\frac{30 \times 4 \times 1.5 \times 10^{4}}{3 \times 10^{4}}}=\sqrt{60}=7.74,
$$

While calculating the bearing construction (the second task), the oriented module was chosen orthogonally which consists of the space frame having four vertical posts at the edges, two longitudinal beams and seven cross beams that are completely made of metal.

On the surface of the frame steel flooring for the carriageway with the supporting longitudinal and cross edges was placed.

The load-bearing frame of orthogonal module represents special construction consisting of longitudinal and cross beams. Its elements are subjected to complex stress condition: compression, bend and torsion. In this regard, the cross-sections of structural elements are determined from the thin-wall pipes framed with four angular elements. This is necessary for strengthening the construction. Geometrical sizes of the frame elements were selected taking into account the compliance with requirements of traffic organization and norms of road automotive industry.

Load-bearing constructions of the frame for ensuring space rigidity and stability are tied by longitudinal and transversal binding constructions. The dynamic effect due to vehicle driving is considered by the introduction of dynamic coefficient $K d$ that is determined by experimental method. For this construction, $K d=1.3$.

For providing conditions of durability, rigidity and stability of the bearing elements of the unified module, calculation of carriageway plate and elements of the space frame on the vertical useful load according to Euronorm requirements was done ${ }^{2}$. Next, we present calculation of the module in the following sequence.

The rectangular plate with size $L \times B$ in the plan from above represents constructively orthotropic plate supported with cross edges with the pace $L / 6$ and longitudinal edges with the pace $B / 4$.

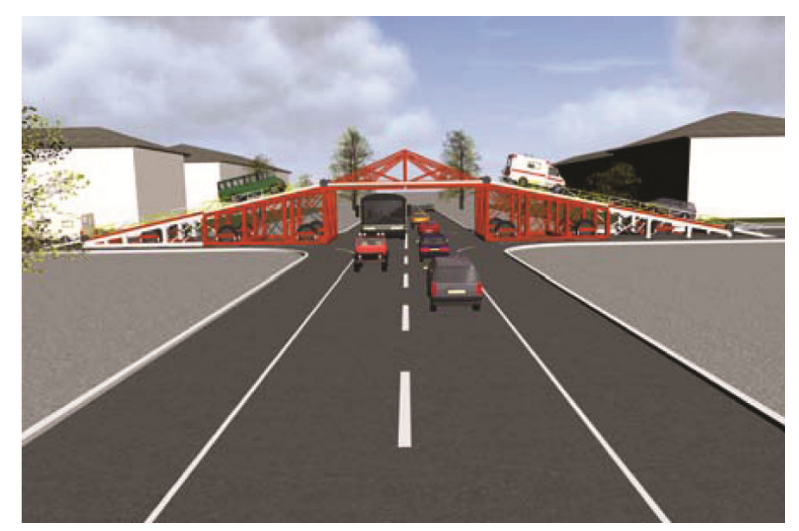

Figure 2. Mobile overcrossing.

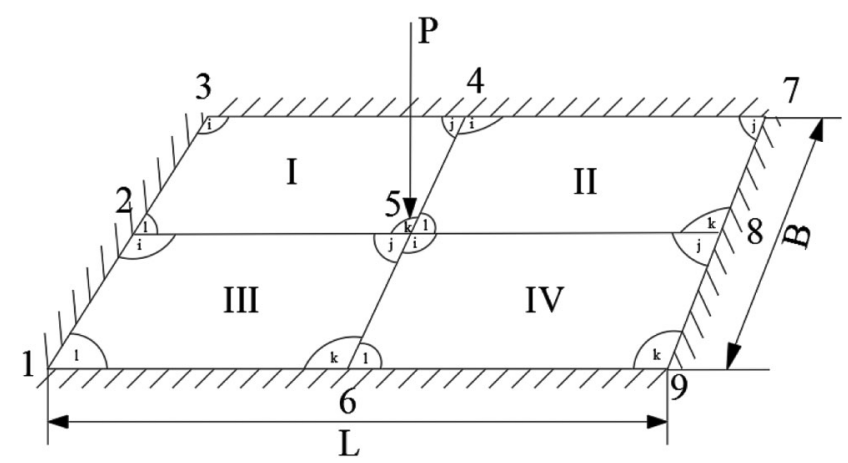

Figure 3. Calculated scheme of the plate using method of finite elements. 
Table 1. Solution matrix of the method of finite differences

\begin{tabular}{|c|c|c|c|c|c|c|c|c|c|c|c|c|}
\hline & 1 & 2 & 3 & 4 & 5 & 6 & 7 & 8 & 9 & 10 & 11 & 12 \\
\hline 1 & $a_{1}+a_{5}+a_{6}$ & $a_{2}$ & $a_{5}$ & 0 & $a_{3}$ & 0 & 0 & 0 & 0 & 0 & 0 & 0 \\
\hline 2 & $a_{2}$ & $a_{1}+a_{6}$ & $a_{2}$ & $a_{5}$ & 0 & $a_{3}$ & $a_{4}$ & 0 & 0 & 0 & 0 & 0 \\
\hline 3 & $a_{5}$ & $a_{2}$ & 0 & $a_{2}$ & 0 & 0 & $a_{3}$ & $a_{4}$ & 0 & 0 & 0 & 0 \\
\hline 4 & 0 & $2 a_{5}$ & 0 & 0 & 0 & 0 & 0 & $a_{3}$ & 0 & 0 & 0 & 0 \\
\hline 5 & $a_{3}$ & $a_{4}$ & 0 & 0 & $a_{1}+a_{5}+a_{6}$ & $a_{2}$ & $a_{5}$ & 0 & $a_{3}$ & $a_{4}$ & 0 & 0 \\
\hline 6 & $a_{4}$ & $a_{3}$ & $a_{4}$ & 0 & 0 & 0 & $a_{2}$ & $a_{5}$ & 0 & $a_{3}$ & $a_{4}$ & 0 \\
\hline 8 & 0 & 0 & 0 & $a_{3}$ & 0 & 0 & 0 & 0 & 0 & 0 & 0 & $a_{3}$ \\
\hline 9 & $2 a_{6}$ & 0 & 0 & 0 & 0 & 0 & 0 & 0 & 0 & 0 & $a_{5}$ & 0 \\
\hline 10 & 0 & $2 a_{6}$ & 0 & 0 & 0 & 0 & 0 & 0 & $a_{2}$ & 0 & $a_{2}$ & $a_{5}$ \\
\hline 11 & 0 & 0 & 0 & 0 & 0 & 0 & 0 & 0 & $a_{5}$ & $a_{2}$ & 0 & $a_{2}$ \\
\hline 12 & 0 & 0 & 0 & 0 & 0 & 0 & 0 & 0 & 0 & 0 & 0 & 0 \\
\hline
\end{tabular}

For universality of calculated expressions, the following geometrical and rigidity characteristics provided to parameters of vertical posts have been accepted

$$
\begin{aligned}
& \alpha_{2}=\frac{l_{2}}{l_{1}} ; \quad \alpha_{3}=\frac{l_{3}}{l_{1}} ; \quad g_{2}=\frac{E J_{2}}{E J_{1}} ; \\
& g_{2 k}=\frac{E J_{2 k}}{E J_{2}} ; \quad g_{3}=\frac{E J_{3}}{E J_{1}} ; \quad g_{3 k}=\frac{E J_{3 k}}{E J_{1}},
\end{aligned}
$$

where $l_{i}$ are the lengths of post elements $(i=1,2,3) ; E J_{i}$ their flexural rigidity; $G J_{i}$ the rotating rigidities, and $\alpha_{2}$, $\alpha_{3}$ are the dimensionless ratio of lengths of longitudinal and cross beams of the frame to its length.

Calculation for durability and rigidity of the plate is performed by two numerical methods: method of finite differences (MFD) and method of finite elements (MFE). Boundary conditions include jamming the plate along its outline s.4. $^{3,4}$.

Calculation by MFD was carried out at density of the grid $\left(n_{x} \times n_{y}\right)$ and using the MathCad program ${ }^{5}$.

Calculation using MFE was performed by dividing the plate surface into four rectangular elements.

Taking into account the double symmetry (on axes $x$ and $y$ ), the number of unknown movements is 12 for MFD and 3 for MFE. Comparison showed good agreement of results using both numerical methods. This proves the reliability of the received values.

In case of MFD, grids were used for manual $(4 \times 3)$ and machine $(4 \times 3)$ calculation. For MFE, a $2 \times 2$ grid was used for manual calculation (Figure 3 ).

For $M_{x}$ (bending moment on $x$-axis), $M_{y}$ (bending moment on $y$-axis) and $M_{x y}$ (torsion moment) of the plate, the corresponding axial stresses $\sigma_{x}, \sigma_{y}, \tau_{x y}$ were determined and durability of the plate was checked ${ }^{6}$

$\sigma_{x}=\frac{6 M_{x}}{t^{2}} ; \sigma_{y}=\frac{6 M_{y}}{t^{2}} ; \tau_{x y}=\frac{6 M_{x y}}{t^{2}} ; K d\left(\sigma_{\max }\right) \leq R$, where $t$ is the flooring thickness $(20 \mathrm{~mm}) ; \sigma_{\max }$ the largest actual tension; $K d(=1.3)$ the dynamic coefficient and $R$ is the calculated resistance of steel (AUSS 09Г2C) at the bend $(300 \mathrm{MPa})$. Also, the condition of rigidity (on deflections) was as follows ${ }^{7}$

$$
\left(\frac{1}{W_{\max }}=\frac{1}{2150}\right)<\left[\frac{1}{W}=\frac{1}{1000}\right]
$$

where $W_{\max }$ is the maximal deflection of the plate and $[1 / W]$ is the plate deflection allowed according to norms.

The system of the simple algebraic equations, on the basis of MFD can be represented as follows

$$
A \cdot \vec{w}=\vec{R}_{p}
$$

where $\vec{w}$ is the vector of unknown node movements; $\vec{R}_{p}$ the vector of the free members considering the load acting on the plate and $A$ is the square matrix of order $n$ (Table 1).

As an example, the plate of size $(L \times B)=(7.5 \times 3.5 \mathrm{~m})$ is calculated, at density of the grid $\left(n_{x} \times n_{y}\right)=(8 \times 6)$.

The following values of deflections in grid nodes are received

$$
\begin{aligned}
& W_{1}=\frac{0.809}{D_{x}} ; \quad W_{2}=\frac{1.079}{D_{x}} ; \quad W_{3}=\frac{1.145}{D_{x}} ; \quad W_{4}=\frac{1.151}{D_{x}} ; \\
& W_{5}=\frac{1.744}{D_{x}} ; \quad W_{6}=\frac{2.401}{D_{x}} ; \quad W_{7}=\frac{2.578}{D_{x}} ; \quad W_{8}=\frac{2.604}{D_{x}} ; \\
& W_{9}=\frac{2.122}{D_{x}} ; \quad W_{10}=\frac{2.497}{D_{x}} ; \quad W_{11}=\frac{3.175}{D_{x}} ; \\
& W_{12}=\frac{3.212}{D_{x}} ; \quad W_{\max }=\frac{3.212}{D_{x}},
\end{aligned}
$$




$$
D_{x}=\frac{E t^{3}}{12\left(1-V^{2}\right)} .
$$

Calculation of the load-bearing frame of the orthogonal module was performed by precise analytical method of displacements.

Figure 4 shows the calculated scheme of the frame taking into account the double symmetry.

$$
A \cdot \vec{z}+\vec{R}_{p}=0,
$$

where $A$ is the square matrix of the 11th order (Table 2).

The total number of unknown angular and linear movements of four nodes of the frame $(A, B, C, D)$ is equal to $11\left(z_{i}=1,2, \ldots, 11\right)^{9-11}$.

Canonical equation of the method of displacements can be represented as follows

$$
z_{k_{1}} Z+z_{k_{2}} Z+\ldots+z_{k_{11}} Z+R_{k p}=0
$$

where $k=1,2, \ldots n$.

\section{Discussion}

After calculation of unknown node displacements, the epure can be calculated by the formula

$$
M=\left(\sum_{k=1}^{11} M_{k} z_{k}\right)+M_{p} .
$$

The epure of transverse $(Q)$ and longitudinal forces $(N)$ is calculated using the usual theory of constructions ${ }^{7-10}$.

As a test task considering Table 2, calculation of space frame having the following data is performed

$$
L=7.2 \mathrm{~m} ; \quad B=3.5 \mathrm{~m} ; l_{1}=H=4.7 \mathrm{~m} ; l_{2}=\frac{L}{6}=1.2 \mathrm{~m} ;
$$

$l_{3}=\frac{B}{4}=0.875 \mathrm{~m} ; \quad \alpha_{2}=\frac{1.2}{4.7}=0.255 ; \quad \alpha_{3}=\frac{0.875}{4.7}=0.186 ;$

$$
\begin{aligned}
& E J_{1}=16.35 \times 10^{6}\left(\mathrm{~N} \times \mathrm{m}^{2}\right) ; \quad G_{1 . k}=0.022 \times 10^{6} ; \\
& E J_{2}=61.8 \times 10^{6}\left(\mathrm{~N} \times \mathrm{m}^{2}\right) ; \quad G_{2 . k}=0.1367 \times 10^{6} ; \\
& E J_{3}=19.86 \times 10^{6}\left(\mathrm{~N} \times \mathrm{m}^{2}\right) ; \quad G_{3 . k}=0.0799 \times 10^{6} ; \\
& g_{2}=3.780 ; \quad g_{2 . k}=0.00836 ; \quad g_{3}=1.2147 ; \\
& g_{3 . k}=0.0049 .
\end{aligned}
$$

According to these, data values of nodal movements (Figure 4) are calculated

$$
\begin{aligned}
& z_{1}=\frac{0.527}{i_{0}} ; \quad z_{2}=\frac{0.487}{i_{0}} ; \quad z_{3}=\frac{-0.101}{i_{0}} ; \quad z_{4}=\frac{0.939}{i_{0}} ; \\
& z_{5}=\frac{-0.12}{i_{0}} ; \quad z_{6}=\frac{0.53}{i_{0}} ; \quad z_{7}=\frac{0.015}{i_{0}} ; \quad z_{8}=\frac{1.139}{i_{0}} ; \\
& z_{9}=\frac{0.342}{i_{0}} ; \quad z_{10}=\frac{0.024}{i_{0}} ; \quad z_{11}=\frac{0.135}{i_{0}} .
\end{aligned}
$$

On the basis of results of force calculation (Figures 58), the stressed condition of the frame was studied, and durability and stability of its elements were checked.

As cross-sections of elements of the frame, the complex section consisting of pipes and framing angles (Figure 10) is used.
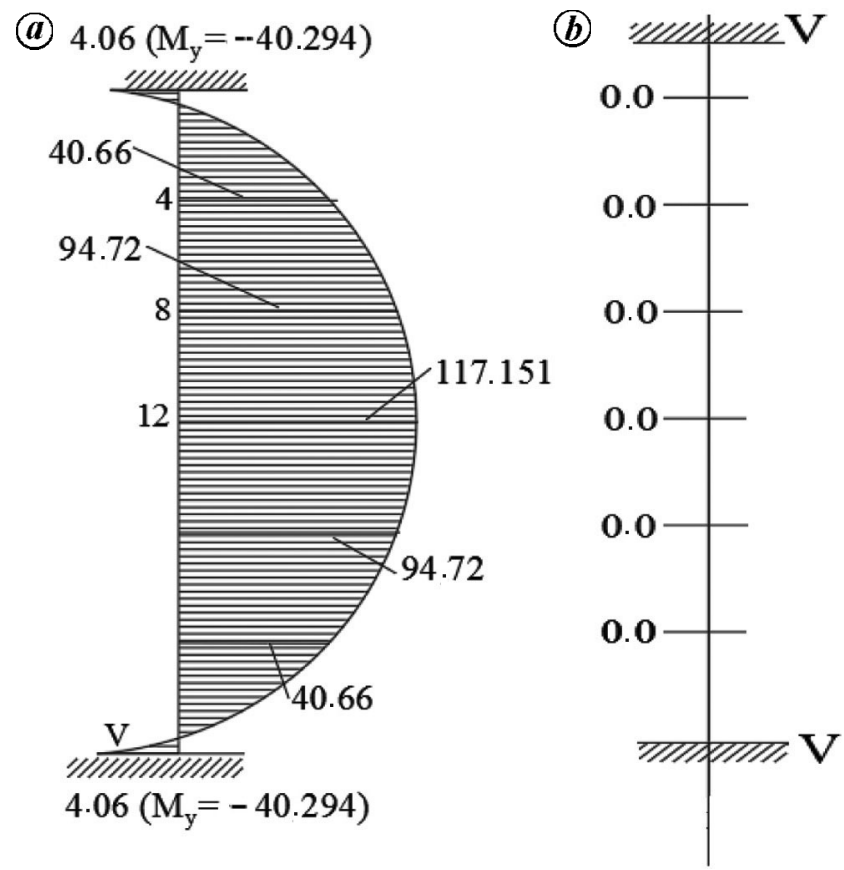

Figure 4. Epures: $\boldsymbol{a}, M_{y}(\mathrm{kNm}) ; \boldsymbol{b}, M_{x y}(\mathrm{kNm})$.

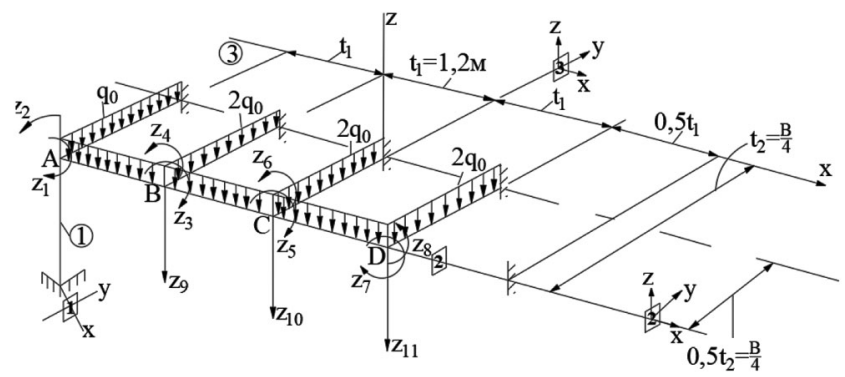

Figure 5. Calculated scheme of the space frame. 


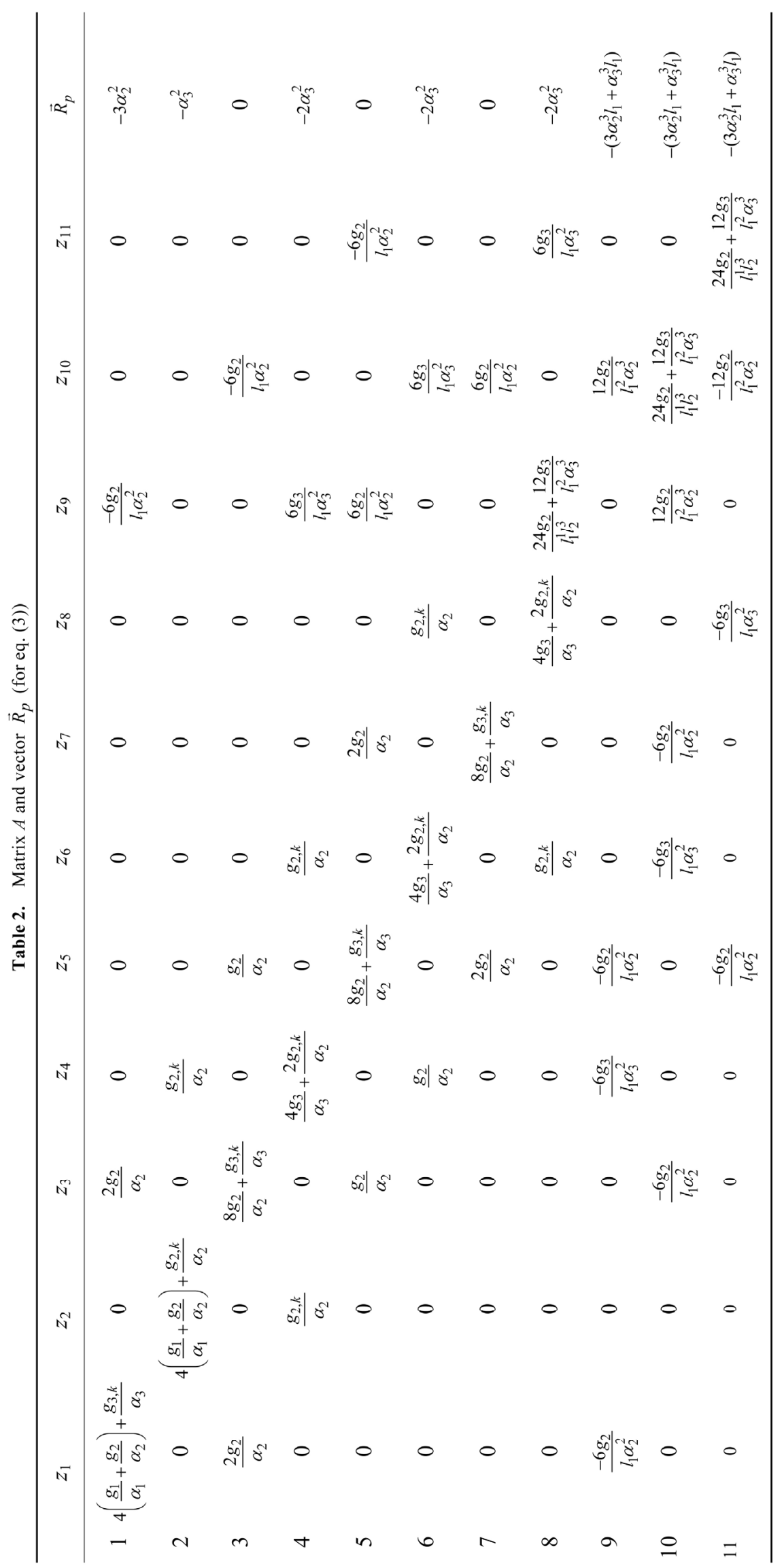


Realization durability and stability conditions of the space frame elements were determined according to the theory of mechanics of materials (strength of materials). It

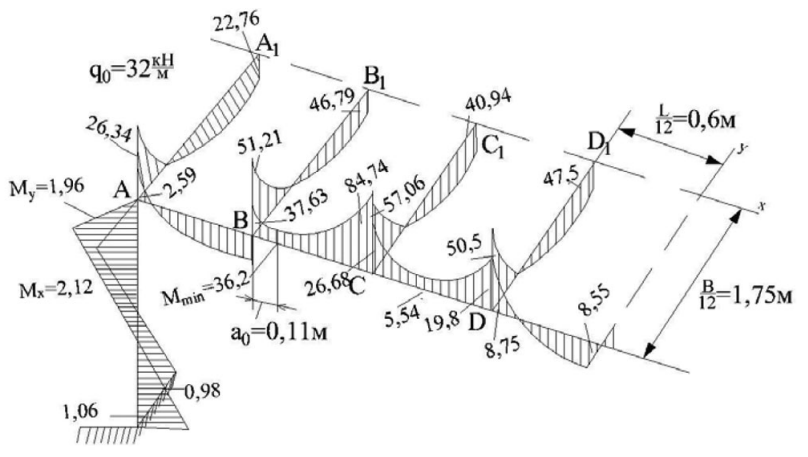

Figure 6. Calculated epure of deflection moments.

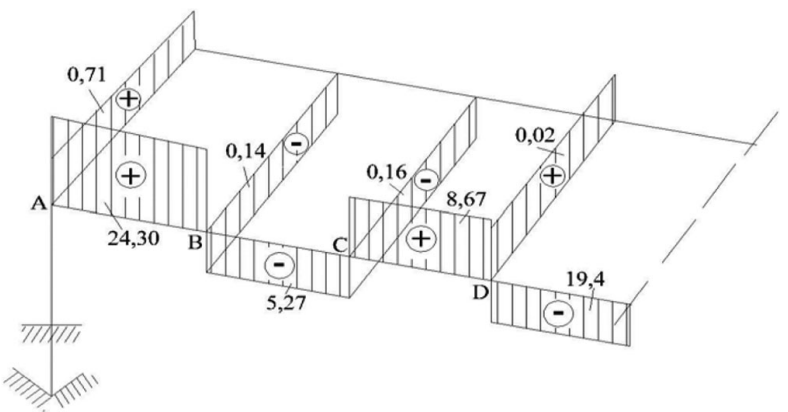

Figure 7. Calculated epure of torques.

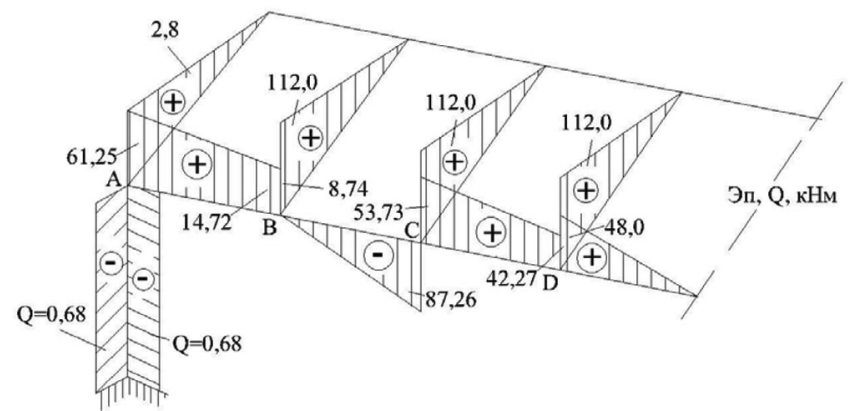

Figure 8. Calculated epure of transversal forces.

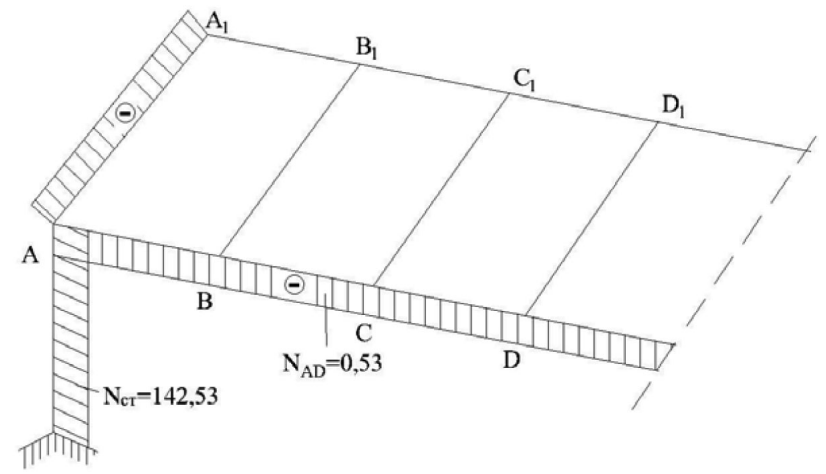

Figure 9. Calculated epure of longitudinal forces. has been taken into account that the elements of frame construction are subjected to complex resistance: compression, bend and torsion ${ }^{12-14}$.

(a) Checking the durability conditions of vertical posts (taking into account flexible work of steel)

$$
K d\left[\left(\frac{N}{A R}\right)^{3 / 2}+\frac{M_{x}}{W_{x} \times R}+\frac{M_{y}}{W_{y} \times R}\right] \leq 1,
$$

where $K d(=1.3)$ is the dynamic coefficient; $R=$ $300 \mathrm{MPa}$ is the calculated resistance of steel (AUSS 09Г2C); $A, W_{x}, W_{y}$ are the area and resistance moments of transverse sections respectively ${ }^{15-17}$.

(b) Checking the stability of the whole post in the plane of action of the moment $M=M_{x}$

$$
K d\left(\frac{N}{\varphi_{x}^{B H} \times A}\right) \leq R_{b},
$$

where $\varphi_{x}^{B H}=f\left(\lambda_{x}, m_{1 x}\right)$ is the coefficient of the longitudinal bending.

(c) Checking the stability of the whole post from the plane of action of the moment of $M=M_{x}$

$$
K d\left(\frac{N}{c \varphi_{y} A}\right) \leq R,
$$

where $c \varphi_{y}$ is the coefficient of space stability.

(d) Checking the durability conditions of longitudinal and cross beams (by the third failure theory) ${ }^{15-17}$.

$$
\begin{aligned}
& K d\left(\sigma_{\text {cе } ~}=\frac{N}{A}+\frac{M_{\text {экв }}}{W_{x}}\right)=R, \\
& M_{r d}=\sqrt{M_{u}^{2}+M_{k}^{2}},
\end{aligned}
$$

where $M_{u}$ is the bending moment and $M_{k}$ is the torque moment.

(e) Checking the durability of longitudinal and crossbeams for cross-section

$$
\left(\tau=\frac{Q \times S_{1}}{J_{x} \times \delta_{C T}}\right) K d \leq R_{c p},
$$

where $Q$ is the transversal force and $R_{c p}(=130 \mathrm{MPa})$ is the calculated resistance for cross-section.

The solution of eqs (7) and (9) was carried out using the standard MathCad program.

\section{Conclusion}

The construction of a mobile overcrossing is discussed in this study that allows us to solve the problem of traffic jams on roads. The calculation of all load-bearing elements of the construction of the overcrossing is carried 


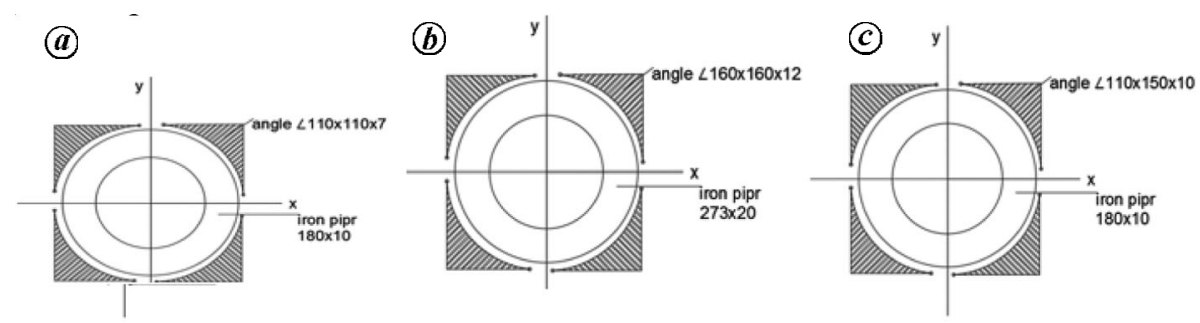

Figure 10. Transverse sections of elements of the space frame: $\boldsymbol{a}$, vertical posts; $\boldsymbol{b}$, longitudinal posts; $c$, cross posts.

out. The calculation of the plate of the carriageway is done by MFD and MFE. The reliability of the results has also been proved.

The accepted concrete geometrical and physicalmechanical characteristics of load-bearing constructions and the supporting edges of steel flooring (plate) with large drift provide their durability.

The calculation of special frame is carried out by the precise analytical method of displacements. This method allows us to calculate any frame with various geometrical and rigidity properties, which proves the universality of the method. On the basis of the results, the concentration of the overcrossing has been done. The technical drawings of the overcrossing in accordance to all standard requirements have also been worked out.

1. Montgomery, D. C., Introduction to Statistical Quality Control, Wiley, 2012, 7th edn, p. 768.

2. Altintas, Y., Manufacturing Automation, Metal Cutting Mechanics, Machine Tool Vibrations, and CNC Design, Cambridge University Press, 2012, col. 12, no. 6, pp. 13-14.

3. Tasci, L. and Kuloglu, N., Investigation of a new transition curve. Baltic J. Road Bridge Eng., 2011, 6(1), 23-29.

4. Ziatdinov, R., Yoshida, N. And Kim, T.-W., Analytic parametric equations of log-aesthetic curves in terms of incomplete gamma functions. Comput. Aided Geometr. Design, 2012, 29(2), 129-140.

5. Gordeev, V. N. and Perelmuter, A. V., Loads and Impacts on Buildings and Structures, ACB Pub., Moscow, 2009, p. 528.

6. Balabekova, K. G. and Kadyrov, A. S., Research work mobile overpass. In Proceedings of the International scientific-practical conference 'Integration of science, education and production basis for the implementation of the Plan of Nation: selected papers' (ed. Gazaliev A. M.), Karaganda, Kazakhstan, 10-11 December 2015, KSTU, pp. 241-243.

7. Hobbacher, A., Recommendations for fatigue design of welded joints and components. International Institute of Welding, NY, USA, 2009; doc.XIII-1965r14-03/XV-1127r14-03.

8. Hu, J., Duncan, J. and Marciniak, Z., Mechanics of Sheet Metal Forming, Butterworth-Heinemann, 2010, p. 211.

9. Bituminous, P. B. and Ritche. J. Appl. Chem., 1953, N3.

10. Bridge Construction Equipment, 2013, p. 488.

11. Mechanics of Sheet Metal Forming, 2010, p. 211.

12. Nikolaĭ Nikolaevich Zorev, Metal cutting mechanics, 1966, p. 526.

13. Bogdanov, B. I. and Vladimirsky, S. R., Design of bridges and pipes. In Metal Bridges, Route Pub., Moscow, 2005, p. 460.

14. Chmielewski, R. and Wolniewicz, A., Classification of the modernized DMS-65 folded bridge structure according to STANAG 2021 requirements. Roads and Bridges - Drogi i Mosty, 16, 2, 2017, pp. 147-158; doi:10.7409/rabdim.017.010.

15. Ahmadiev, S. K., Theoretical Foundations Design Mechanical Engineer, KSTU Pub., Karaganda, 2010, p. 99.

16. Kadyrov, A. S. and Kadyrova, I. A., Basics of scientific research. Karaganda, KSTU, 2015, p. 267.

17. Ganyukov, A. A. and Balabekova, K. G., Calculation of the Spatial Frame of the Modular Overpass, University Proceedings. PSU, 2016, No. 4, pp. 63-68.

Received 5 April 2017; revised accepted 8 September 2018

doi: $10.18520 / \mathrm{cs} / \mathrm{v} 116 / 19 / 1544-1550$ 